IOANNIS K. ARgyros (Lawton, OK)

\title{
A NEW APPROACH FOR FINDING WEAKER CONDITIONS FOR THE CONVERGENCE OF NEWTON'S METHOD
}

Abstract. The Newton-Kantorovich hypothesis (15) has been used for a long time as a sufficient condition for convergence of Newton's method to a locally unique solution of a nonlinear equation in a Banach space setting. Recently in [3], [4] we showed that this hypothesis can always be replaced by a condition weaker in general (see (18), (19) or (20)) whose verification requires the same computational cost. Moreover, finer error bounds and at least as precise information on the location of the solution can be obtained this way. Here we show that we can further weaken conditions (18)-(20) and still improve on the error bounds given in [3], [4] (see Remark 1(c)).

1. Introduction. In this study we are concerned with the problem of approximating a locally unique solution $x^{*}$ of the equation

$$
F(x)=0,
$$

where $F$ is a Fréchet-differentiable operator defined on an open subset $D$ of a Banach space $X$ with values in a Banach space $Y$.

A large number of problems in applied mathematics and also in engineering are solved by finding solutions of certain equations. For example, dynamical systems are mathematically modeled by difference or differential equations, and their solutions usually represent states of the systems. For the sake of simplicity, assume that a time-invariant system is driven by the equation $\dot{x}=G(x)$ (for some suitable operator $G$ ), where $x$ is the state. Then the equilibrium states are determined by solving equation (1). Similar equations are used in the case of discrete systems. The unknowns of engineering equations can be functions (difference, differential, and integral equations),

2000 Mathematics Subject Classification: 65H10, 65G99, 47H17, 49M15.

Key words and phrases: Newton's method, Banach space, majorant principle, NewtonKantorovich hypothesis/theorem, Fréchet derivative, Lipschitz, center-Lipschitz condition. 
vectors (systems of linear or nonlinear algebraic equations), or real or complex numbers (single algebraic equations with single unknowns). Except in special cases, the most commonly used solution methods are iterative - when starting from one or several initial approximations a sequence is constructed that converges to a solution of the equation. Iteration methods are also applied for solving optimization problems. In such cases, the iteration sequences converge to an optimal solution of the problem at hand. Since all of these methods have the same recursive structure, they can be introduced and discussed in a general framework.

The most popular method for generating a sequence $x_{n}$ approximating $x^{*}$ is undoubtedly Newton's method given by

$$
x_{n+1}=x_{n}-F^{\prime}\left(x_{n}\right)^{-1} F\left(x_{n}\right) \quad(n \geq 0)\left(x_{0} \in D\right) .
$$

Here $F^{\prime}\left(x_{n}\right)$ denotes the Fréchet derivative of the operator $F$ evaluated at $x=x_{n}$ [2], [5], [8]. The geometric interpretation of Newton's method is well known if $F$ is a real function. In such a case $x_{n+1}$ is the point where the line $y-F\left(x_{n}\right)=F^{\prime}\left(x_{n}\right)\left(x-x_{n}\right)$ tangent to the graph of $F(x)$ at the point $\left(x, F\left(x_{n}\right)\right)$ intersects the $x$-axis.

Consider the Lipschitz condition

$$
\left\|F^{\prime}\left(x_{0}\right)^{-1}\left(F^{\prime}(x)-F^{\prime}(y)\right)\right\| \leq \ell\|x-y\|
$$

for all $x, y \in D$, and some $\ell \geq 0, x_{0} \in D$ such that $F^{\prime}\left(x_{0}\right)^{-1} \in L(Y, X)$, the space of bounded linear operators from $Y$ into $X$. Using (3) we can arrive at the famous Newton-Kantorovich condition (14) which is sufficient for the convergence of Newton's method (2).

A survey of local and semilocal convergence results for Newton's method (2) can be found in [1], [2], [5], [6], [8], [10], [11], and the references there.

Recently in [3], [4] by using a combination of (3) and the center-Lipschitz condition

$$
\left\|F^{\prime}\left(x_{0}\right)^{-1}\left(F^{\prime}(x)-F^{\prime}\left(x_{0}\right)\right)\right\| \leq \ell_{0}\left\|x-x_{0}\right\| \quad(x \in D),
$$

we challenged (15) and showed that this hypothesis can always be replaced by the weaker (18) or (19) or (20) whose verification requires the same computational cost. Moreover finer error bounds on the distances $\| x_{n+1}-$ $x_{n}\|,\| x_{n}-x^{*} \|(n \geq 0)$ and at least as precise information on the location of the solution were given. Note that in general $0 \leq \ell_{0} \leq \ell$ and $\ell / \ell_{0}$ can be arbitrarily large [3].

Here we show that we can further weaken conditions (18)-(20) and still improve on error bounds given in [3], [4] (see Remark 1(c)).

2. Semilocal convergence analysis for Newton's method (2). It is convenient to define a scalar iteration $\left\{t_{n}\right\}(n \geq 0)$ for some given $\eta \geq 0$, 
$\ell_{0} \geq 0, \ell \geq 0$ by

$$
t_{0}=0, \quad t_{1}=\eta, \quad t_{n+2}=t_{n+1}+\frac{\ell\left(t_{n+1}-t_{n}\right)^{2}}{2\left(1-\ell_{0} t_{n+1}\right)} \quad(n \geq 0) .
$$

It plays a crucial role in the study of the convergence of Newton's method (2). It turns out that under certain conditions $\left\{t_{n}\right\}$ is a majorizing sequence for $\left\{x_{n}\right\}$.

If

$$
\ell_{0} t_{n}<1 \quad \text { for all } n \geq 0\left(\ell_{0} \neq 0\right),
$$

then it follows from (5) that $\left\{t_{n}\right\}$ is nondecreasing and bounded above by $\ell_{0}^{-1}$, and as such it converges to some $t^{*} \in\left[0,1 / \ell_{0}\right]$. Below we provide conditions which imply (6).

We need the following general result on majorizing sequences for Newton's method (2).

Lemma 1. Assume there exist constants $d \geq 0, \eta \geq 0, \ell_{0} \geq 0, \ell \geq 0$, and sequences $1>a_{n} \geq 0, b_{n} \geq 0, c_{n} \geq 0, \bar{d}_{n}$, and $d_{n} \geq 0$ such that for

$$
\begin{aligned}
& \bar{d}_{0}=d_{0}=0, \bar{d}_{1}=d_{1}=\eta, a_{n}=\ell_{0} \bar{d}_{n}, b_{n}=\frac{1}{1-a_{n}}, c_{n}=\ell b_{n}, \\
& \bar{d}_{n}=t_{1}+\frac{c_{1}}{2}\left(t_{1}-t_{0}\right)^{2}+\frac{c_{2}}{2}\left(t_{2}-t_{1}\right)^{2}+\cdots+\frac{c_{n-1}}{2}\left(t_{n-1}-t_{n-2}\right)^{2} \\
& \quad(n \geq 2)
\end{aligned}
$$

the following conditions hold for all $n \geq 0$ :

$$
\bar{d}_{n} \leq d_{n} \leq d<\ell_{0}^{-1} .
$$

Then the sequence $\left\{t_{n}\right\}(n \geq 0)$ generated by (5) is nondecreasing, bounded above by $\ell_{0}^{-1}$ and converges to some $t^{*} \in\left[0,1 / \ell_{0}\right]$.

Moreover the following estimates hold:

$$
t_{n} \leq \bar{d}_{n} \quad(n \geq 0),
$$

and

$$
t_{n+1}-t_{n}=\frac{c_{n}}{2}\left(t_{n}-t_{n-1}\right)^{2} \quad(n \geq 1) .
$$

Proof. It suffices to show that the hypotheses of the lemma imply condition (6). Indeed using (5), (7)-(9) we can have in turn for all $n \geq 2$ (since (6) holds for $n=0,1$ by the initial conditions):

$$
\begin{aligned}
t_{n+2} & \leq t_{n+1}+\frac{c_{n+1}}{2}\left(t_{n+1}-t_{n}\right)^{2} \\
& \leq t_{n}+\frac{c_{n}}{2}\left(t_{n}-t_{n-1}\right)^{2}+\frac{c_{n+1}}{2}\left(t_{n+1}-t_{n}\right)^{2} \\
& \leq \cdots \leq t_{1}+\frac{c_{1}}{2}\left(t_{1}-t_{0}\right)^{2}+\cdots+\frac{c_{n+1}}{2}\left(t_{n+1}-t_{n}\right)^{2} \\
& =\bar{d}_{n+2} \leq d_{n+2},
\end{aligned}
$$


which shows (10) for all $n \geq 0$. That is, by (9) and (12) condition (6) holds. Moreover by (5), (7) and (8) we obtain (11).

That completes the proof of Lemma 1.

We can provide some special choices of parameters and sequences defined above.

Remark 1. (a) Assume

$$
\ell_{0}=\ell
$$

for

$$
\left\|F^{\prime}\left(x_{0}\right)^{-1} F\left(x_{0}\right)\right\| \leq \eta,
$$

and

$$
h=2 \ell \eta \leq 1 .
$$

Note that condition (14) is the famous Newton-Kantorovich hypothesis which is sufficient for the convergence of Newton's method (2) to $x^{*}$ [2], [5], [6], [8], [11]. Define $d_{n}, d(n \geq 0)$ by

$$
d_{n}=\eta+\frac{1}{2^{1}} h^{2^{1}-1} \eta+\cdots+\frac{1}{2^{n-1}} h^{2^{n-1}-1} \eta
$$

and

$$
d=\frac{1-\sqrt{1-2 \ell \eta}}{\ell} .
$$

Then it follows from the proof of the Newton-Kantorovich theorem that $a_{n}<1$. Moreover conditions (6) and (9) hold.

(b) Assume that the following conditions hold:

$$
h_{\delta}=\left(\delta \ell_{0}+\ell\right) \eta \leq \delta \quad \text { for } \delta \in[0,1]
$$

or

$$
h_{\delta} \leq \delta, \quad \frac{2 \ell_{0} \eta}{2-\delta} \leq 1, \quad \frac{\ell_{0} \delta^{2}}{2-\delta} \leq \ell \quad \text { for } \delta \in[0,2)
$$

or

$$
h_{\delta} \leq \delta, \quad \ell_{0} \eta \leq 1-\frac{1}{2} \delta \quad \text { for } \delta \in\left[\delta_{0}, 2\right)
$$

where

$$
\delta_{0}=\frac{-\ell / \ell_{0}+\sqrt{\left(\ell / \ell_{0}\right)^{2}+8 \ell / \ell_{0}}}{2} .
$$

Then by Theorem 2 in [3, p. 311] condition (9) holds for

$$
d_{n}=\eta+\frac{\delta}{2} \eta+\cdots+\left(\frac{\delta}{2}\right)^{n+1} \eta
$$


and

$$
d=\frac{2 \eta}{2-\delta}
$$

In [3], [4] we showed that in general conditions (18)-(20) are weaker than (15). Set e.g. $\delta=1$ in (18).

Moreover if $\left\{s_{n}\right\}$ denotes the sequence $\left\{t_{n}\right\}$ when $\ell=\ell_{0}$ we showed for all $n \geq 0$ :

$t_{n} \leq s_{n}, \quad t_{n+1}-t_{n} \leq s_{n+1}-s_{n} t_{n} \leq s_{n}, \quad t^{*} \leq s^{*}=\lim _{n \rightarrow \infty} s_{n}, \quad t^{*}-t_{n} \leq s^{*}-s_{n}$.

Note that strict inequality holds in the first two bounds if $\ell_{0}<\ell$ for all $n \geq 1$. Other possible choices exist (see, e.g., Lemma 2).

(c) So far we showed that if (15) and (18) or (19) or (20) hold then by directly comparing the majorizing sequence $\left\{t_{n}\right\}$ with $\left\{s_{n}\right\}$ we see that the former is finer (more precise) in case $\ell_{0}<\ell$. However if (15) is violated and (18) or (19) or (20) hold then estimate (22) does not guarantee the quadratic convergence of $\left\{t_{n}\right\}$. Note that in this case we cannot even compare the two majorizing sequences, since only the convergence of $\left\{t_{n}\right\}$ is guaranteed. In order to rectify this in Lemma 2 we provide conditions (see (24)) similar to (18)-(20) which however guarantee the quadratic convergence of the majorizing sequence $\left\{t_{n}\right\}$. Finally, note that the limit $t^{*}$ of the majorizing sequence $\left\{t_{n}\right\}$ is at least as small as the limit $s^{*}$ of $\left\{s_{n}\right\}$. That is, the information on the uniqueness ball of the solution $x^{*}$ is at least as precise under our approach.

REMARK 2. In case $\ell_{0}=0$ the convergence of (5) is guaranteed provided that $\ell \eta / 2 \in[0,1)$, since $0 \leq t_{n+2}-t_{n+1}<t_{n}-t_{n-1}$ for $n \geq 0$.

Next we show how to find conditions for the convergence of the majorizing sequence $\left\{t_{n}\right\}$.

Remark 3. Assume that there exist parameters $\ell_{0}>0, \ell>0, \eta>0$, $a \geq 1$ such that

$$
p_{a}=\left(\ell+2 \ell_{0} a\right) \eta<2 .
$$

Then

$$
I=\left[1, \frac{1}{\ell_{0} \eta}-\frac{\ell}{2 \ell_{0}}\right] \neq \emptyset
$$

the function

$$
c=c(a)=\frac{\ell}{2\left(1-\ell_{0} a \eta\right)}
$$

is well defined on $I$, and

$$
0 \leq c \eta<1
$$


Moreover assume that

$$
t_{n+1} \leq a \eta \quad \text { for all } n \geq 0 .
$$

It then follows that

$$
t_{n+2}-t_{n+1}=\frac{\ell}{2\left(1-\ell_{0} t_{n+1}\right)}\left(t_{n+1}-t_{n}\right)^{2} \leq c\left(t_{n+1}-t_{n}\right)^{2}
$$

and

$$
c\left(t_{n+2}-t_{n+1}\right) \leq\left[c\left(t_{n+1}-t_{n}\right)\right]^{2} \leq \cdots \leq(c \eta)^{2^{n+1}} .
$$

Let

$$
d(a)=\eta+\frac{1}{c}\left[(c \eta)^{2^{1}}+\cdots+(c \eta)^{2^{n}}+\cdots\right] .
$$

Then $d$ is a well defined function for all $a \in I$. Finally, assume that there exists $\beta \in I$ such that

$$
d(\beta) \leq \beta \eta .
$$

It then follows that $d(\beta)$ is an upper bound on the sequence $\left\{\bar{d}_{n}\right\}$. That is,

$$
t_{n} \leq \bar{d}_{n} \leq d(\beta) \text {. }
$$

Consequently, under hypotheses (24) and (31) the sequence $\left\{t_{n}\right\}$ is nondecreasing and bounded above by $d(\beta)$ and as such it converges to some $t^{*} \in\left[\eta, 1 / \ell_{0}\right]$.

Using induction on $n \geq 0$ we can show condition (6), and consequently drop hypothesis (28). Indeed, (6) holds for $n=0,1$ by the initial conditions. By (5) we have

$$
\begin{gathered}
t_{2}-t_{1} \leq c(\beta)\left(t_{1}-t_{0}\right)^{2}, \\
\ell_{0} t_{2} \leq \ell_{0}\left[\eta+c(\beta)\left(t_{1}-t_{0}\right)^{2}\right] \leq \ell_{0} d(\beta) \leq \ell_{0} \beta \eta<1,
\end{gathered}
$$

and since $t_{n+1}-t_{n} \leq c(\beta)\left(t_{n}-t_{n-1}\right)^{2}$, we get

$\ell_{0} t_{n+1} \leq \ell_{0}\left[\eta+c(\beta)\left(t_{1}-t_{0}\right)^{2}+\cdots+\left(c(\beta)\left(t_{n}-t_{n-1}\right)^{2}\right] \leq \ell_{0} d(\beta) \leq \ell_{0} \beta \eta<1\right.$, which completes the induction.

Hence we showed:

LEMMA 2. Under the stated hypotheses:

(a) condition (6) holds;

(b) the sequence $\left\{t_{n}\right\}$ is nondecreasing and converges to some $t^{*}$ such that

$$
t_{n} \leq t^{*} \leq \frac{1}{\ell_{0}} \quad\left(\ell_{0} \neq 0\right)
$$


(c) the following error bounds hold for all $n \geq 0$ :

$$
\begin{gathered}
0 \leq t_{n+2}-t_{n+1} \leq c(\beta)\left(t_{n+1}-t_{n}\right)^{2}, \\
0 \leq t^{*}-t_{n} \leq c(\beta)^{-1} \gamma_{n}, \\
\gamma_{n}=\lim _{k \rightarrow \infty}\left\{[c(\beta) \eta]^{2^{n+k-1}}+\cdots+[c(\beta) \eta]^{2^{n}}\right\} \\
\leq \lim _{k \rightarrow \infty} \frac{[c(\beta) \eta]^{2^{n}}\left[1-(c(\beta) \eta)^{2 k}\right]}{1-[c(\beta) \eta]^{2}} \leq \frac{[c(\beta) \eta]^{2^{n}}}{1-[c(\beta) \eta]^{2}} .
\end{gathered}
$$

REMARK 4. (a) The existence of $\beta$ is guaranteed by the intermediate value theorem provided there exist $\beta_{0}, \beta_{1} \in I$ with $\beta_{0}<\beta_{1}$ such that

$$
f\left(\beta_{0}\right) f\left(\beta_{1}\right)<0,
$$

where the function $f$ is given by

$$
f(a)=d(a)-a \eta .
$$

Other existence conditions for finding zeros $\beta$ of a scalar function $f$ can be found in the literature [2], [5], [6], [8], [9].

(b) It follows from (30) that condition (31) can be replaced by the stronger but easier to check

$$
d^{0}(\beta) \leq \beta \eta
$$

or

$$
d^{1}(\beta) \leq \beta \eta
$$

where

$$
\begin{aligned}
& d^{0}(a)=\frac{1}{c(a)\left[1-(c(a) \eta)^{2}\right]}, \\
& d^{1}(a)=\eta+\frac{c(a) \eta^{2}}{1-(c(a) \eta)^{2}} .
\end{aligned}
$$

Below is the main semilocal convergence theorem for Newton's method (2) using Lipschitz condition (3), center-Lipschitz condition (4), and condition (6):

Theorem 1. Let $F: D \subseteq X \rightarrow Y$ be a Fréchet-differentiable operator. Assume that conditions (3), (4), (6), (14) hold and

$$
\bar{U}\left(x_{0}, 1 / \ell_{0}\right)\left\{x \in X \mid\left\|x-x_{0}\right\| \leq 1 / \ell_{0}\right\} \subseteq D \quad \text { for } \ell_{0} \neq 0 .
$$

Then the sequence $\left\{x_{n}\right\}(n \geq 0)$ generated by Newton's method (2) is well defined, remains in $\bar{U}\left(x_{0}, t^{*}\right)$ for all $n \geq 0$ and converges to a solution $x^{*} \in \bar{U}\left(x_{0}, t^{*}\right)$ of equation $F(x)=0$. Moreover, the following error bounds 
hold for all $n \geq 0$ :

$$
\begin{aligned}
\left\|x_{n+2}-x_{n+1}\right\| & \leq \frac{\ell\left\|x_{n+1}-x_{n}\right\|^{2}}{2\left[1-\ell_{0}\left\|x_{n+1}-x_{0}\right\|\right]} \leq t_{n+2}-t_{n+1}, \\
\left\|x_{n}-x^{*}\right\| & \leq t^{*}-t_{n}
\end{aligned}
$$

where the iteration $\left\{t_{n}\right\}(n \geq 0)$ is given by (5). The solution $x^{*}$ is unique in $\bar{U}\left(x_{0}, t^{*}\right)$ provided that

$$
\ell_{0} t^{*}<1
$$

Furthermore, if there exists $R>t^{*}$ such that

$$
U\left(x_{0}, R\right) \subseteq D
$$

and

$$
\ell_{0}\left(t^{*}+R\right) \leq 2,
$$

then the solution $x^{*}$ is unique in $U\left(x_{0}, R\right)$.

Proof. Let us prove that

$$
\left\|x_{k+1}-x_{k}\right\| \leq t_{k+1}-t_{k}
$$

and

$$
\bar{U}\left(x_{k+1}, t^{*}-t_{k+1}\right) \subseteq \bar{U}\left(x_{k}, t^{*}-t_{k}\right)
$$

for all $k \geq 0$. For every $z \in \bar{U}\left(x_{1}, t^{*}-t_{1}\right)$,

$$
\left\|z-x_{0}\right\| \leq\left\|z-x_{1}\right\|+\left\|x_{1}-x_{0}\right\| \leq t^{*}-t_{1}+t_{1}=t^{*}-t_{0}
$$

implies $z \in \bar{U}\left(x_{0}, t^{*}-t_{0}\right)$. Since also

$$
\left\|x_{1}-x_{0}\right\|=\left\|F^{\prime}\left(x_{0}\right)^{-1} F\left(x_{0}\right)\right\| \leq \eta=t_{1}-t_{0},
$$

(51) and (52) hold for $k=0$. Given they hold for $n=0,1, \ldots, k$, then

$$
\left\|x_{k+1}-x_{0}\right\| \leq \sum_{i=1}^{k+1}\left\|x_{i}-x_{i-1}\right\| \leq \sum_{i=1}^{k+1}\left(t_{i}-t_{i-1}\right)=t_{k+1}-t_{0}=t_{k+1}
$$

and

$$
\left\|x_{k}+\theta\left(x_{k+1}-x_{k}\right)-x_{0}\right\| \leq t_{k}+\theta\left(t_{k+1}-t_{k}\right)<t^{*}, \quad \theta \in[0,1] .
$$

Using (2) we obtain the approximation

$$
\begin{aligned}
F\left(x_{k+1}\right) & =F\left(x_{k+1}\right)-F\left(x_{k}\right)-F^{\prime}\left(x_{k}\right)\left(x_{k+1}-x_{k}\right) \\
& =\int_{0}^{1}\left[F^{\prime}\left(x_{k}+\theta\left(x_{k+1}-x_{k}\right)\right)-F^{\prime}\left(x_{k}\right)\right]\left(x_{k+1}-x_{k}\right) d \theta
\end{aligned}
$$


and by (3),

$$
\begin{aligned}
& \left\|F^{\prime}\left(x_{0}\right)^{-1} F\left(x_{k+1}\right)\right\| \\
& \quad \leq \int_{0}^{1}\left\|F^{\prime}\left(x_{0}\right)^{-1}\left[F^{\prime}\left(x_{k}+\theta\left(x_{k+1}-x_{k}\right)\right)-F^{\prime}\left(x_{k}\right)\right]\right\| d \theta\left\|x_{k+1}-x_{k}\right\| \\
& \quad \leq \frac{\ell}{2}\left\|x_{k+1}-x_{k}\right\|^{2} \leq \frac{\ell}{2}\left(t_{k+1}-t_{k}\right)^{2} .
\end{aligned}
$$

It follows from (4) and (6) that

$$
\left\|F^{\prime}\left(x_{0}\right)^{-1}\left[F^{\prime}\left(x_{k+1}\right)-F^{\prime}\left(x_{0}\right)\right]\right\| \leq \ell_{0}\left\|x_{k+1}-x_{0}\right\| \leq \ell_{0} t_{k+1}<1,
$$

and the Banach Lemma on invertible operators [8] shows that the inverse $F^{\prime}\left(x_{k+1}\right)^{-1}$ exists and

$$
\left\|F^{\prime}\left(x_{k+1}\right)^{-1} F^{\prime}\left(x_{0}\right)\right\| \leq \frac{1}{1-\ell_{0}\left\|x_{k+1}-x_{0}\right\|} \leq \frac{1}{1-\ell_{0} t_{k+1}} .
$$

Therefore, by (2), (5), (55) and (56) we obtain in turn

$$
\begin{aligned}
\left\|x_{k+2}-x_{k+1}\right\| & =\left\|F^{\prime}\left(x_{k+1}\right)^{-1} F\left(x_{k+1}\right)\right\| \\
& \leq\left\|F^{\prime}\left(x_{k+1}\right)^{-1} F^{\prime}\left(x_{0}\right)\right\| \cdot\left\|F^{\prime}\left(x_{0}\right)^{-1} F\left(x_{k+1}\right)\right\| \\
& \leq \frac{\ell\left\|x_{k+1}-x_{k}\right\|^{2}}{2\left(1-\ell_{0}\left\|x_{k+1}-x_{0}\right\|\right)} \leq \frac{\ell\left(t_{k+1}-t_{k}\right)^{2}}{2\left(1-\ell_{0} t_{k+1}\right)} \\
& =t_{k+2}-t_{k+1} .
\end{aligned}
$$

Thus for every $z \in \bar{U}\left(x_{k+2}, t^{*}-t_{k+2}\right)$ we have $\left\|z-x_{k+1}\right\| \leq\left\|z-x_{k+2}\right\|+\left\|x_{k+2}-x_{k+1}\right\| \leq t^{*}-t_{k+2}+t_{k+2}-t_{k+1}=t^{*}-t_{k+1}$. That is,

$$
z \in \bar{U}\left(x_{k+1}, t^{*}-t_{k+1}\right) .
$$

Estimates (57) and (58) imply that (51) and (52) hold for $n=k+1$. By induction the proof of (51) and (52) is complete.

From (6), (51) and (52), $\left\{x_{n}\right\}(n \geq 0)$ becomes a Cauchy sequence, and as such it converges to some $x^{*} \in \bar{U}\left(x_{0}, t^{*}\right)$ (since $\bar{U}\left(x_{0}, t^{*}\right)$ is a closed set) such that

$$
\left\|x^{*}-x_{k}\right\| \leq t^{*}-t_{k} .
$$

The combination of (56) and (57) yields $F\left(x^{*}\right)=0$. 
Finally, to show uniqueness let $y^{*}$ be a solution of the equation $F(x)=0$ in $U\left(x_{0}, R\right)$. It follows from (4), the estimate

$$
\begin{aligned}
\| F^{\prime}\left(x_{0}\right)^{-1} & \int_{0}^{1}\left[F^{\prime}\left(y^{*}+\theta\left(x^{*}-y^{*}\right)\right)-F^{\prime}\left(x_{0}\right)\right] \| d \theta \\
& \leq \ell_{0} \int_{0}^{1}\left\|y^{*}+\theta\left(x^{*}-y^{*}\right)-x_{0}\right\| d \theta \\
& \leq \ell_{0} \int_{0}^{1}\left[\theta\left\|x^{*}-x_{0}\right\|+(1-\theta)\left\|y^{*}-x_{0}\right\|\right] d \theta<\frac{\ell_{0}}{2}\left(t^{*}+R\right) \leq 1,
\end{aligned}
$$

and the Banach Lemma on invertible operators that the linear operator

$$
L=\int_{0}^{1} F^{\prime}\left(y^{*}+\theta\left(x^{*}-y^{*}\right)\right) d \theta
$$

is invertible. Using the identity

$$
0=F\left(x^{*}\right)-F\left(y^{*}\right)=L\left(x^{*}-y^{*}\right)
$$

we deduce $x^{*}=y^{*}$. That completes the proof of Theorem 1 .

REMARK 5. (a) $1 / \ell_{0}$ can be replaced by $t^{*}$ in condition (45).

(b) If $\ell_{0}=0$ condition (45) can be replaced by

$$
U\left(x_{0}, t^{*}\right) \subseteq D .
$$

Hypothesis (6) is then replaced by

$$
\ell n / 2 \in[0,1)
$$

(see also Remark 2), whereas condition (50) is not needed.

We complete this study with a simple numerical example.

Example 1 . Let $X=Y=\mathbb{R}, x_{0}=-.6, D=[-1,2]$, and define a function $F$ on $D$ by

$$
F(x)=\frac{1}{3} x^{3}+.0897462 .
$$

Using (3), (4), (14) and (62) we obtain

$$
\eta=.049295, \quad \ell_{0}=3 . \overline{8}, \quad \ell=11 . \overline{1} .
$$

Condition (15) is violated, since

$$
h=1.095 \overline{4}>1 .
$$

Therefore the Newton-Kantorovich theorem cannot guarantee that Newton's method starting from $x_{0}=-.6$ converges to $x^{*}=-.645722284$. Our condition (24) for say $\beta=1.5$ holds since

$$
p_{\beta}=1.228305<2 \text {. }
$$


Moreover, we obtain

$$
d(\beta)=.0711047<.0739425=\beta \eta,
$$

which shows that (31) is satisfied. That is, our Theorem 1 guarantees the convergence of Newton's method to $x^{*}$.

\section{References}

[1] J. Appell, E. De Pascale and P. P. Zabrejko, On the application of the NewtonKantorovich method to nonlinear integral equations of Urysohn type, Numer. Funct. Anal. Optim. 12 (1991), 271-283.

[2] I. K. Argyros, Advances in the Efficiency of Computational Methods and Applications, World Sci., River Edge, NJ, 2000.

[3] - On the convergence and application of generalized Newton methods, Nonlinear Stud. 10 (2003), 307-321.

[4] -, On the Newton-Kantorovich hypothesis for solving nonlinear equations, J. Comput. Appl. Math. 169 (2004), 315-332.

[5] I. K. Argyros and F. Szidarovszky, The Theory and Applications of Iteration Methods, CRC Press, Boca Raton, FL, 1993.

[6] J. E. Dennis, Toward a unified convergence theory for Newton-like methods, in: Nonlinear Functional Analysis and Applications, L. B. Rall (ed.), Academic Press, New York, 1971, 425-472.

[7] M. J. Gutiérrez, M. A. Hernández and M. A. Salanova, Accessibility of solutions by Newton's method, Internat. J. Comput. Math. 57 (1995), 239-247.

[8] L. V. Kantorovich and G. P. Akilov, Functional Analysis, Pergamon Press, Oxford, 1982.

[9] A. M. Ostrowski, Solution of Equations in Euclidean and Banach Spaces, Academic Press, New York, 1973.

[10] F. A. Potra and V. Pták, Sharp error bounds for Newton's process, Numer. Math. 34 (1980), 67-72.

[11] T. A. Yamamoto, A method for finding sharp error bounds for Newton's method under the Kantorovich assumptions, ibid. 49 (1986), 203-220.

Department of Mathematical Sciences

Cameron University

Lawton, OK 73505, U.S.A.

E-mail: ioannisa@cameron.edu

Received on 11.1.2005;

revised version on 5.10.2005 